\title{
RANDOM NOISE ASSESSMENT IN AERIAL AND SATELLITE IMAGES
}

\author{
Irina Anikeeva ${ }^{1 *}$, Aleksandr Chibunichev ${ }^{2}$ \\ ${ }^{1}$ JSC "Roscartography”, Moscow, Russia - ia.anikeeva@ roscartography.ru \\ ${ }^{2}$ Moscow State University of Geodesy and Cartography, Moscow, Russia - agchib@miigaik.ru
}

KEY WORDS: Aerial and Satellite Images, Aerial and Satellite Image Quality Assessment, Random Image Noise, Harmonic Analysis, Accuracy Assessment

\begin{abstract}
:
Random noise in aerial and satellite images is one of the factors, decreasing their quality. The noise level assessment in images is paid not enough attention. The method of numerical estimation of random image noise is considered. The object of the study is the image noise estimating method, based on harmonic analysis. The capability of using this method for aerial and satellite image quality assessment is considered. The results of the algorithm testing on model data and on real satellite images with different terrain surfaces are carried out. The accuracy estimating results for calculating the root-mean-square deviation (RMS) of random image noise by the harmonic analysis method are shown.
\end{abstract}

\section{INTRODUCTION}

Image's quality in terms of it's visual perception is one of the most important characteristics. It directly affects the quality and volume of information transmitted by images about terrain objects.

There are many factors that affect the aerial and satellite image's quality, which are determined both by surveying conditions (cloud cover, haze, illumination, etc.) and by technical means of obtaining and processing images.

Among factors, reducing the image's quality is digital noise, which appear in image as randomly located pixels with significantly different brightness or color from average brightness or color of neighboring pixels (Khryashchev, 2011; Lapshenkov, 2012).

Random noise affects the interpretation quality of aerial and satellite images, that's why its level must be determined when performing production works to assess the suitability of this data for creating the final geospatial products based by them. This task also arises when performing testing and validation of remote sensing satellite systems that are being put into operation. The most noticeable effect of random noise on interpretation image quality becomes when detecting low-contrast objects and objects that fall into the shadow of high-rise structures, an example is shown in Figure 1.

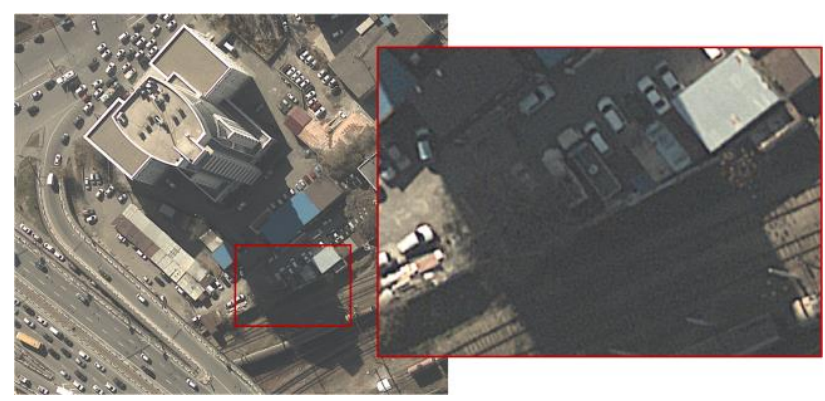

Figure 1. An example of losses in objects and their details, that fall into the shadow, due to the image random noise

The causes of random image noise are both camera specifications, such as physical pixel size, matrix sensitivity, temperature, etc., and surveying settings, as the value of the exposure (Zotov, 2015).

There is a number of publications devoted to determining the aerial and satellite image noise level topic (Beloglazov et al., 2009; Schowengerdt, 2010, Yi et al., 2018, Lim et al., 2018), but in general, this problem is not given enough attention, and in practice, the noise level indicator is not always being assessed when evaluating the images quality, despite its obvious influence on interpretation properties. Sufficient number of researches are devoted to random noise removing to improve the images quality (Donoho, 1995; Lin, S. and Xiaosu, G., 2020), but this is not the purpose of this study, due that these algorithms unavoidably affect the geometric properties of an image.

The performance of aerial and satellite surveys is associated with assessing the quality of obtained materials, that carried out at images primary post-processing stage. Currently, the images quality assessment is executed visually by the operator. This method allows only a qualitative image's assessment by its brightness and contrast characteristics, and color balance, taking into account the peculiarities of the operator's psychophysical perception. The subjectivity of such assessment is beyond doubt. In this way it is not possible to estimate the noise level, which is often visually noticeable in the image. The statement about the random image noise presence without determining its quantitative assessment only based on visual analysis has a few worth.

Figure 2.a shows a piece of an image scene, obtained by Canopus-B spacecraft, and an image, obtained by airborne camera DMC II in Figure 2.b. Both images show a visually noticeable random noise.

* Corresponding author 

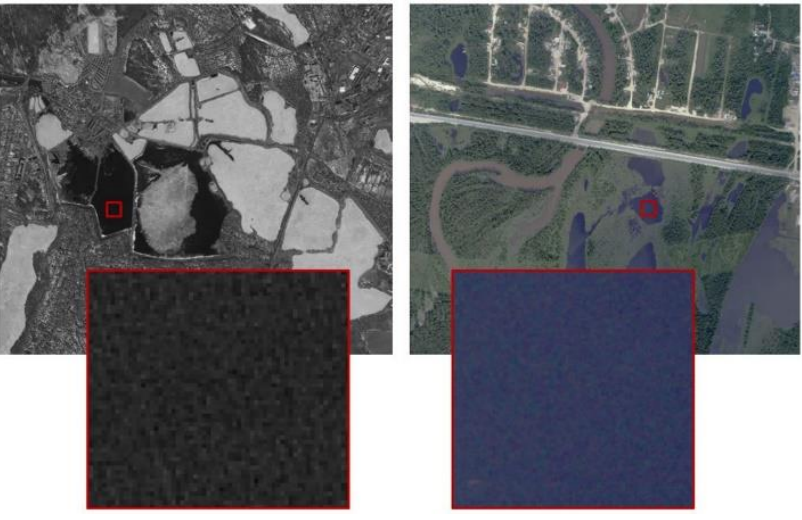

Figure 2.a) Random noise on an image scene, obtained by Canopus-B spacecraft

Figure 2.b) Random noise on an image obtained by airborne mapping camera DMC II

There are various ways to assess the image noise and there are a lot of research work devoted to this issue, which describe the algorithms and perform their comparative analysis (Samoilin, 2006; Chen et al, 2019; Lapshenkov, 2012; and others)

There is a number of publications devoted to determining the aerial and satellite image noise level topic (Beloglazov et al, 2009; Schowengerdt, 2010), but in general, this problem is not given enough attention, and in practice, the noise level indicator is not evaluated at all when assessing the images quality, despite its obvious influence on interpretation properties.

Lapshenkov $(2012,2013)$ proposed an algorithm for assessing the image noise level based on harmonic analysis. The author shows the advantage of the proposed algorithm in comparison with alternative methods, and presents the experimental results, in which the algorithm based on harmonic analysis allows to obtain the most accurate assessing of the random image noise level.

The purpose of the study is to test the reliability of the image noise estimation algorithm based on harmonic analysis and the possibility of its application for assessing the aerial and satellite images quality, obtained for mapping purposes.

\section{METHODOLOGY}

To test the proposed noise estimation algorithm and the possibility of its application for assessing the aerial and satellite images there are several tasks to be solved: to estimate the accuracy of the algorithm on the model data; to estimate the algorithm's resistance to the type (contour load) of the underlying surface; to analyse the obtained results and evaluate the algorithm's accuracy.

The signal-to-noise ratio is used as a measure of the noise impact on image's quality. It is known different ways to evaluate this indicator. Shovengerdt (2010) gives the following methods:

the signal-to-noise contrast ratio (1) as an "amplitude» estimate or the squared contrast ratio can be used to estimate the noise «power» (2):

$$
\begin{gathered}
S / N_{\text {amplitude }}=\frac{\mathrm{C}_{\text {signal }}}{\mathrm{C}_{\text {noise }}} \\
S / N_{\text {power }}=\left(S / N_{\text {amplitude }}\right)^{2}=\left(\frac{\mathrm{C}_{\text {signal }}}{\mathrm{C}_{\text {noise }}}\right)^{2}
\end{gathered}
$$

where: $\quad C_{\text {signal }}=$ contrast value of signal

$C_{\text {noise }}=$ contrast value of noise .
The noise influence on image's quality also can be assessed as the standard deviations (RMS) ratio of signal and noise levels:

$$
S / N_{R M S}=\frac{\sigma_{\text {signal }}}{\sigma_{\text {noise }}}
$$

or as a variance ratio:

$$
S / N_{\text {variance }}=\frac{\sigma_{\text {signal }}^{2}}{\sigma_{\text {noise }}{ }^{2}}
$$

where: $\quad \sigma_{\text {signal }}(S)=$ RMS of pixel values for the image signal $\sigma_{\text {noise }}\left(N_{R M S}\right)=$ RMS of pixel values for the image noise.

Often, the logarithm of the signal-to-noise ratio is used as a random noise level indicator in decibels (Lapshenkov, 2012; Schowengerdt, 2010):

$$
S / N_{d B}=10 \cdot \lg \left(S / N_{\text {power }}\right)=20 \cdot \lg \left(S / N_{R M S}\right)
$$

The image noise estimation method, proposed by Lapshenkov, allows to determine the random noise RMS $\left(N_{R M S}\right)$ based on harmonic analysis, so it is expedient to use the formula (3) to calculate the signal-to-noise ratio.

In Lapshenkov $(2012,2013)$ the implementation of methods for estimating the image noise, including those based on harmonic analysis, in the MATLAB is presented. The proposed method is based on the analysis of spectral power density distribution of the signal, describing the image. This distribution is calculated using the two-dimensional Fourier transform. In this article, the implementation of proposed method for estimation image random noise is performed in Python 3.x. The program code of the respective function is shown below.

\section{def NOISE(image):}

\#calculating the image size:

rows = image.shape[0] \#vertical in pixels

cols $=$ image. shape[1] \#horizontal in pixels

\# working number of samples for the two-dimensional Fourier transform:

$\mathrm{n}=2 * *(\mathrm{np} \cdot \log 2$ (rows) $)$ \#vertical

$\mathrm{m}=2 * *(\mathrm{np} . \log 2(\mathrm{cols}))$ \#horizontal

I = np.fft.fft2(image) \#two-dimensional Fourier transform of an image

$\mathrm{I}=\mathrm{abs}(\mathrm{I})$ \#calculating the absolute values of the Fourier image $\mathrm{I}[0,0]=0$ \#removing the constant component

\#selecting the quantization step:

$\mathrm{p}=0,02 \mathrm{\# a}$ priori specified parameter that determines the accuracy of the noise level calculating, the smaller this parameter, the higher the accuracy of the calculations; however, reducing this parameter leads to increase in the amount of calculations

$\mathrm{q}=\mathrm{p} * \operatorname{np} \cdot \operatorname{sqrt}((\mathrm{n} * \mathrm{~m}) * * 2 /(\mathrm{m} * \mathrm{n}-1))$ \#calculating the quantization step size of the FFT results

\#quantizing the FFT results for histogram creation:

$\mathrm{I}=\mathrm{np} \cdot \operatorname{round}(\mathrm{I} / \mathrm{q}, 0)$

\#selecting the working range for the histogram: max_I = np.ceil(np.max $(\mathrm{I}))$ \#calculating the maximum value $\min \_I=$ np.floor(np.min(I)) \#calculating the minimum value $\mathrm{d}=$ max_I - min_I \#calculating the working range of quantized FFT values 
$\mathrm{d}=\operatorname{int}(\mathrm{d} / \mathrm{p})$ \#calculating the quantization step for the working range of quantized FFT values

\section{\#creating a histogram:}

bins $=$ np.array $(\mathrm{np} . \operatorname{arange}(\mathrm{d})$, dtype $=$ int $)$ \#calculating the number of intervals for the histogram

$\mathrm{I}=\mathrm{np} \cdot \operatorname{array}(\mathrm{I} \cdot \operatorname{ravel}())$

HIST $=$ np.histogram(I, bins) [0] \#histogram creating

\#histogram maximum calculating:

HISTmax $=$ np.max $($ HIST $)$

\#calculating the histogram mode as the weighted average of the value $0,7 *$ HISTmax:

index $=$ np.where $($ HIST $>=0.7 *$ HISTmax $)[0]$

hist $=0$

hist_w $=0$

for $\mathrm{i}$ in range(len(index)):

$\mathrm{k}=$ index $[\mathrm{i}]$

hst $=$ HIST $[\mathrm{k}]$

$\mathrm{hstw}=\mathrm{k} *$ HIST $[\mathrm{k}]$

hist $=$ hist + hst

hist $\_$w $=$hist $\_$w + hstw

mode_w = hist_w/hist \#weighted histogram mode

\#calculating the random image noise RMS:

$\mathrm{Nrms}=$ mode_w $*$ np.sqrt(np.pi/2) $* \mathrm{p} * 1,07$ \#index 1,07 is a correction factor

return Nrms

The signal RMS can be calculated by (6), under the assumption that it is uncorrelated with noise:

$$
\sigma_{\text {signal }}=\sqrt{\sigma_{\text {total }}^{2}-N_{R M S}^{2}}
$$

where: $\quad \sigma_{\text {total }}=$ RMS of total pixel values calculated from the image.

\subsection{Estimation the accuracy of the algorithm on the model data}

A sample of test images for the initial verification of the random noise estimation algorithm, based on harmonic analysis, was created. Images were obtained by adding random gaussian noise with different RMS: $N_{R M S}=1,2,3,4,5,10,20,40,80$ to the artificially created gray wedge's original image with a noise RMS $N_{R M S}=0$. The artificial noisy images sample is shown in Figure 3.

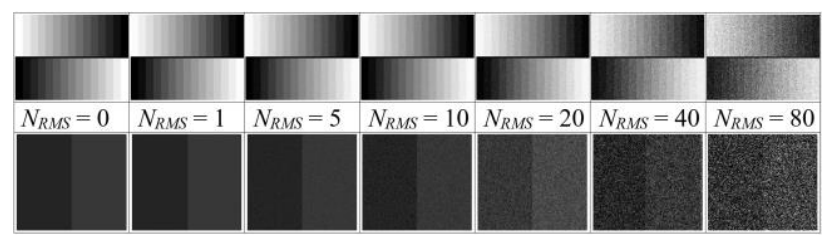

Figure 3. Test sample images, created for the initial verification of the noise level estimation algorithm based on harmonic analysis; the upper row - the overall image's view, the bottom raw - an enlarged part of each image

Table 1 shows the results of random noise assessment $N_{R M S}$ for the model images test sample based on harmonic analysis.

\begin{tabular}{|c|c|c|c|c|c|c|}
\hline \multirow{3}{*}{$\begin{array}{c}\sigma_{\text {total }} \\
\\
N_{\text {RMS }}\end{array}$} & \multirow{3}{*}{  } & \multicolumn{3}{|c|}{$\begin{array}{c}\text { Results, obtained by } \\
\text { the harmonic analysis } \\
\text { method }\end{array}$} & \multirow{2}{*}{\multicolumn{2}{|c|}{$\begin{array}{l}\text { Method's } \\
\text { error }\end{array}$}} \\
\hline & & \multirow{2}{*}{ 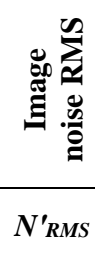 } & \multirow{2}{*}{$\frac{S^{\prime}}{S_{\bar{S}}}$} & \multirow{2}{*}{ 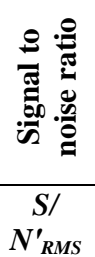 } & & \\
\hline & & & & & $\begin{array}{c}N_{R M S}^{\prime} / \\
N_{R M S}\end{array}$ & $\begin{array}{c}\text { Error } \\
\%\end{array}$ \\
\hline 0 & - & 0,04 & 80,08 & - & 0,96 & 4,00 \\
\hline 1 & 80,08 & 1,13 & 79,99 & 70,79 & 0,94 & 13,00 \\
\hline 2 & 40,04 & 2,04 & 79,89 & 39,16 & 0,99 & 2,00 \\
\hline 3 & 26,69 & 2,98 & 79,79 & 26,78 & 1,01 & 0,67 \\
\hline 4 & 20,02 & 3,89 & 79,70 & 20,49 & 1,02 & 2,75 \\
\hline 5 & 16,02 & 4,85 & 79,60 & 16,41 & 1,03 & 3,00 \\
\hline 10 & 8,01 & 9,52 & 79,10 & 8,31 & 1,05 & 4,80 \\
\hline 20 & 4,00 & 18,27 & 77,85 & 4,26 & 1,09 & 8,65 \\
\hline 40 & 2,00 & 34,50 & 74,26 & 2,15 & 1,15 & 13,75 \\
\hline 80 & 1,00 & 60,75 & 64,82 & 1,07 & 1,31 & 24,06 \\
\hline
\end{tabular}

Table 1. The results of random noise assessment $N_{R M S}$ for the test images sample based on harmonic analysis

Table 1 shows that the harmonic analysis method can provide the image noise RMS assessing with an average absolute error about $7,7 \%$.

Figure 3 shows the graphs of the reference random image noise RMS $\left(N_{R M S}\right)$ for the test sample and random noise RMS, calculated by the harmonic analysis method $\left(N_{R M S}^{\prime}\right)$. Assuming the graphs in Figure 4 and calculations in Table 1, it is obvious that with an increase in the image's noise RMS, the error of its determination also increases.

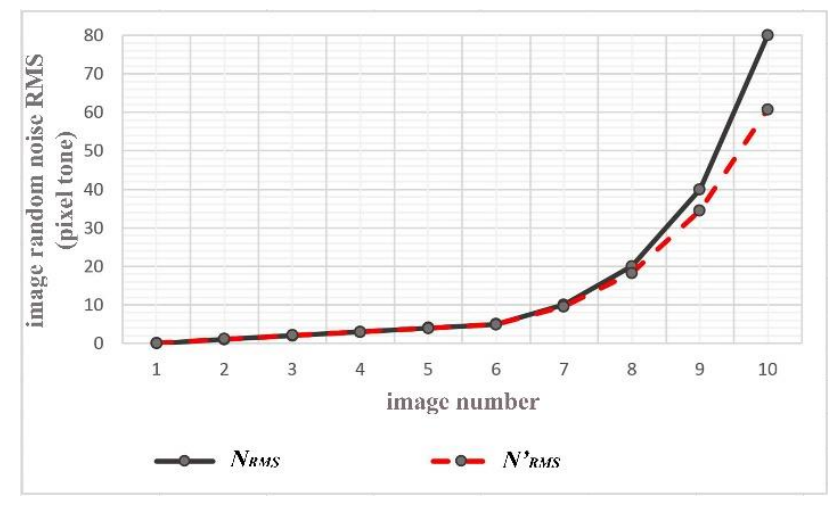

Figure 4. The graphs of the reference random image noise RMS $\left(N_{R M S}\right)$ for the test sample and random noise RMS, calculated by the harmonic analysis method $\left(N_{R M S}^{\prime}\right)$

\subsection{Estimation the algorithm's resistance to the underlying surface type (contour load)}

To estimate the stability of the random noise assessing method, based on harmonic analysis, to the type (contour load) of the underlying surface, a test sample of satellite images with various terrain types was created. There are five main terrain types:
a. urban areas (multistorey buildings),
b. industrial facilities,
c. village settlements,
d. agricultural lands,
e. relief features. 
The images were obtained by Canopus-V spacecraft No. 6 . Sample images are shown in Figure 5.
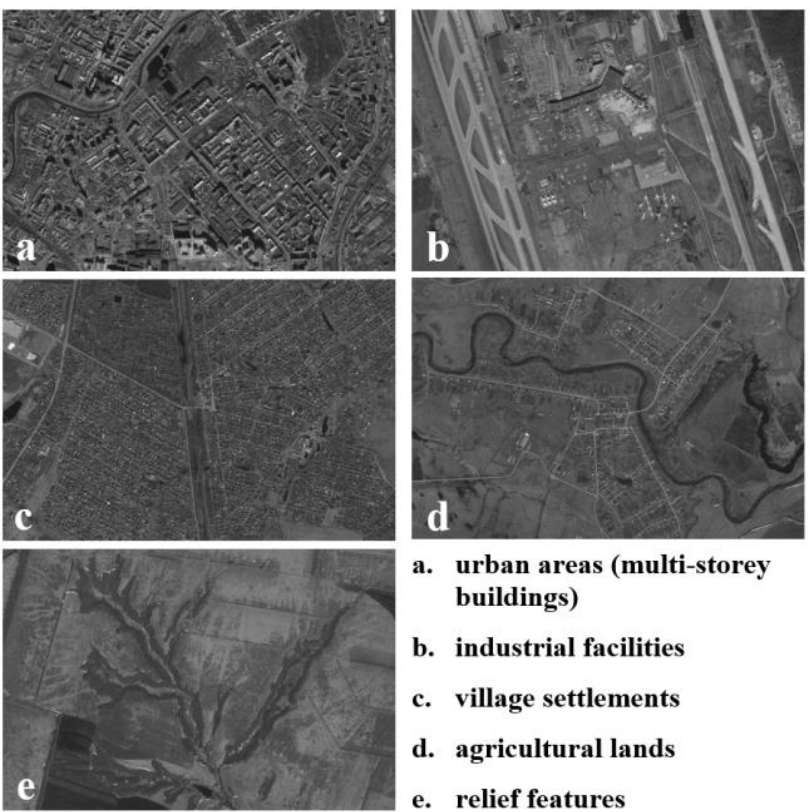

\section{a. urban areas (multi-storey} buildings)

b. industrial facilities

c. village settlements

d. agricultural lands

e. relief features

Figure 5. A sample of images, obtained by the Canopus-V spacecraft, with different terrain types for random noise estimation, based on harmonic analysis

Based on each of these images, a sample, similar to the test sample described above, was created by adding the random gaussian noise with above RMS values. There were 5 samples in total. The noise RMS was estimated taking into account the native image noise of the original image noise $N_{R M S}$ base. The results of random noise assessing based on harmonic analysis for satellite image samples and their accuracy assessment are shown in Table 2.

\begin{tabular}{|c|c|c|c|c|c|}
\hline $\begin{array}{l}\text { Image } \\
\text { sample }\end{array}$ & $\mathbf{a}$ & b & c & d & e \\
\hline$N_{R M S}$ base & 1,77 & 1,41 & 1,76 & 1,45 & 1,40 \\
\hline $\begin{array}{c}\text { Reference } \\
\text { noise RMS }\end{array}$ & \multicolumn{5}{|c|}{$\begin{array}{c}\text { Noise RMS, obtained by the harmonic } \\
\text { analysis method }\end{array}$} \\
\hline$N_{R M S}$ & \multicolumn{5}{|c|}{$N_{R M S}^{\prime}$} \\
\hline $\mathbf{0}$ & 0 & 0 & 0 & 0 & 0 \\
\hline 1 & 1,34 & 1,29 & 1,32 & 1,30 & 1,34 \\
\hline 2 & 2,37 & 2,34 & 2,44 & 2,38 & 2,38 \\
\hline 3 & 3,46 & 3,37 & 3,48 & 3,38 & 3,39 \\
\hline 4 & 4,50 & 4,40 & 4,50 & 4,49 & 4,40 \\
\hline 5 & 5,55 & 5,44 & 5,55 & 5,48 & 5,48 \\
\hline 10 & 10,70 & 10,50 & 10,80 & 10,55 & 10,55 \\
\hline 20 & 20,72 & 20,48 & 20,95 & 20,60 & 20,42 \\
\hline 40 & 38,44 & 39,40 & 38,99 & 39,37 & 39,13 \\
\hline 80 & 65,63 & 68,01 & 66,40 & 66,80 & 68,09 \\
\hline
\end{tabular}

Table 2. The results of random noise assessing based on harmonic analysis for satellite images samples

The results in Table 2 indicate that harmonic analysis method showed a stable result when assessing images with different types of terrain surface. It confirms the method's invariance to this factor and the possibility of it's application for assessing the quality of aerial and satellite images.
2.3 The analysis of the obtained results and evaluation the algorithm's accuracy

Evaluation of the algorithm's accuracy was in calculating the error (in percentage) with which the random image noise RMS can be determined. The accuracy evaluation results are shown in Table 3.

\begin{tabular}{|c|c|c|c|c|c|}
\hline $\begin{array}{c}\text { Image } \\
\text { sample }\end{array}$ & $\mathbf{a}$ & b & c & d & e \\
\hline$N_{R M S}$ base & 1,77 & 1,41 & 1,76 & 1,45 & 1,40 \\
\hline $\begin{array}{c}\text { Reference } \\
\text { noise RMS }\end{array}$ & \multicolumn{5}{|c|}{ Accuracy assessment } \\
\hline NRMS & \multicolumn{5}{|c|}{$N_{R M S}^{\prime} / N_{R M S}$} \\
\hline $\mathbf{0}$ & - & - & - & - & - \\
\hline 1 & 1,34 & 1,29 & 1,32 & 1,30 & 1,34 \\
\hline 2 & 1,19 & 1,17 & 1,22 & 1,19 & 1,19 \\
\hline 3 & 1,15 & 1,12 & 1,16 & 1,13 & 1,13 \\
\hline 4 & 1,13 & 1,10 & 1,13 & 1,12 & 1,10 \\
\hline 5 & 1,11 & 1,09 & 1,11 & 1,10 & 1,10 \\
\hline 10 & 1,07 & 1,05 & 1,08 & 1,06 & 1,06 \\
\hline 20 & 1,04 & 1,02 & 1,05 & 1,03 & 1,02 \\
\hline 40 & 0,96 & 0,99 & 0,97 & 0,98 & 0,98 \\
\hline 80 & 0,82 & 0,85 & 0,83 & 0,84 & 0,85 \\
\hline$N_{R M S}$ & \multicolumn{5}{|c|}{ Error \% } \\
\hline $\mathbf{0}$ & - & - & - & - & - \\
\hline 1 & 34,00 & 29,00 & 32,00 & 30,00 & 34,00 \\
\hline 2 & 18,50 & 17,00 & 22,00 & 19,00 & 19,00 \\
\hline 3 & 15,33 & 12,33 & 16,00 & 12,67 & 13,00 \\
\hline 4 & 12,50 & 10,00 & 12,50 & 12,25 & 10,00 \\
\hline 5 & 11,00 & 8,80 & 11,00 & 9,60 & 9,60 \\
\hline 10 & 7,00 & 5,00 & 8,00 & 5,50 & 5,50 \\
\hline 20 & 3,60 & 2,40 & 4,75 & 3,00 & 2,10 \\
\hline 40 & 3,90 & 1,50 & 2,53 & 1,58 & 2,17 \\
\hline 80 & 17,96 & 14,99 & 17,00 & 16,50 & 14,89 \\
\hline $\begin{array}{c}\text { Mean } \\
\text { value, \%: }\end{array}$ & 13,76 & 11,22 & 13,98 & 12,23 & 12,25 \\
\hline $\begin{array}{c}\text { Total } \\
\text { mean } \\
\text { value, } \% \text { : }\end{array}$ & \multicolumn{5}{|c|}{12,69} \\
\hline
\end{tabular}

Table 3. The algorithm's based on harmonic analysis accuracy evaluation results

The obtained in Table 3 results show that the reviewed method allows to assess the random image noise RMS with an average absolute accuracy about $12,7 \%$.

For random noise assessing in this study the panchromatic images were used. When estimating the noise level of color images, the calculation should be performed in grayscale mode or separately for each color channel, when the detailed analysis is required.

\section{CONCLUSION}

In aerial and satellite image quality assessing the random image noise should be evaluated as one of the important quality characteristic in terms of visual perception. In this study the possibility of the image noise estimation algorithm, based on harmonic analysis, for assessing the aerial and satellite images was tested.

The method's test consisted in the estimation of it's accuracy on the model data. A sample of artificial images with random noise 
RMS of values from 0 to 80 was used. The algorithm showed the results of a high accuracy.

The next step of the study was the algorithm's resistance estimation to the type (contour load) of the underlying surface. Five samples of satellite images with different terrain types were formed for this with random noise addition. The added images noise RMS were the same values, as they were on a previous step for the model data. The obtained results confirmed the stability of a harmonic analysis method to different types of terrain surface and the possibility of it's application for aerial and satellite image quality assessment.

The final step of the study showed, that the harmonic analysis method allows to assess the random image noise RMS with an average absolute accuracy not worse than $13 \%$.

\section{REFERENCES}

Beloglazov, I.N., Veselov, Yu.G., Gulevich, S.P., Danilin, A.A., Karpikov, I.V., 2009: Statistical Noise Characteristics Calculation of a Digital Camera Based on Images Obtained in Flight Conditions. Flight safety issues. 2, 25-30.

Chen, M., Zhang, H., Han, Q., Huang, C.C., 2019: A Convex Nonlocal Total Variation Regularization Algorithm for Multiplicative Noise Removal. EURASIP Journal on Image and Video Processing. doi.org/10.1186/s13640-019-0410-2.

Donoho, D.L., 1995: De-noising by Soft-Thresholding. IEEE Transactions on Information Theory. 41(3), 613-627. doi.org/10.1109/18.382009

Khryashchev, D.A., 2011: About One Method for Determining the Additive Noise Model Most Suitable for the Analyzed Digital Image. Bulletin of the Volgograd State Technical University. 3(76), 24-31.

Lapshenkov E.M., 2012: Non-Reference Assessment of Digital Image Noise Based on Harmonic Analysis. Computer optics. 36(3), 439-447.

Lapshenkov, E.M., 2013: Implementation of Methods for Estimating the Image Noise in MATLAB. Bulletin of the Moscow State University of Instrumentation and Informatics. Instrumentation and information technology. 44, 96-106.

Lim, P.-C., Kim, T., Na, S.-I., Lee, K.-D., Ahn, H.-Y., Hong, J., 2018: Analysis of UAV Image Quality Using Edge Analysis. Int. Arch. Photogramm. Remote Sens. Spatial Inf. Sci., XLII-4, 359364. doi.org/10.5194/isprs-archives-XLII-4-359-2018.

Lin, S., Xiaosu, G., 2020: Analysis of Denoising Interpretation of Remote Sensing Image Based on ICA-Wavelet Ttransform. Int. Arch. Photogramm. Remote Sens. Spatial Inf. Sci., XLII-3W10, 411-414. doi.org/10.5194/isprs-archives-XLII-3-W10411-2020.

Miao, C., 2019: Research on Denoising Processing of Computer Video Electromagnetic Leakage Reduction Image Based on Fuzzy Degree. EURASIP Journal on Image and Video Processing. doi.org/10.1186/s13640-018-0405-4.

Samoilin, E.A., 2006: Algorithms for Impulse Noise Estimating in Problems of Optical Images Digital Filtering. Optical Journal. 73(12), 42-46.

Schowengerdt, R., 2010: Remote Sensing. Models and Methods for Image Processing. Technosphere, Moscow.
Zotov, P.V., 2015: Digital Image Noise and Its Applied Value in Forensic Science. Bulletin of the Saratov State Law Academy. 6(107), 175-179.

Yi, W., Xin, H., Xiafang, Y., Guojun, X., 2018: Analysis of the Image Quality of No Ground Controlled Positioning Precision About Surveying and Mapping Satellite. Int. Arch. Photogramm. Remote Sens. Spatial Inf. Sci., XLII-3, 1821-1825. doi.org/10.5194/isprs-archives-XLII-3-1821-2018. 